sich weder mit Sicherheit erschliessen, wann das Contagium vom Angriffspunkte sich über den Körper ausbreitet, noch ob eine Excision der Initialsclerose und wanm dieselbe vorzunehmen sei.) Mraček.

\title{
Constitutionelle Syphilis-Formen und Beiträge zur Behandlung der Syphilis.
}

Barthélemy. Auto-inoculation des syphilitischen Schankers. (Annales de dermatol. et de syphil. Tom. VI, Nr. 4, p. 200.)

Verfasser glaubt nicht, dass Pontoppidan dorch seine Experimente zur Klärung der Frage über Excision dar Sclerosen beigetragen habe, gibt aber 7.u, dass dadurch bestätigt wurde, was man schon längst wusste, dass nämlich die Syphilis gradation den Körper durchdringt, wie man es z. B. an einer Initialsclerose des Augenlides sehen kann, der die Schwellung präauricularer Drüsen, dann der submaxillaren, der Cervical- und schlirsslich orst der Subclavicular-Drüsen folgt. Barthélemy erinnert ferner an die ähnlichen Versuche von Raynaud und Chauveau, die auch drei Roihen von Impfpusteln mit Vaccine erzeugen konnten, bevor die Immunität im Organismus sich entwickelt hat. Es wird forner ein Fall angeführt, der einen deutlich syphilitischen Initialaffect (chancre syphilitique) und Leistendrüsen zeigte. Die Induration bestand noch nach drei Monaten and die Drüsensehwellungen noch nach vier Monaten, ohne dass sich weitere Syphiliserscheinungen selbst nach 18 Monaten nachweisen liessen. Eine allgomeine Behandlung wurde nicht angewendet. Nun fragt Barthélemy, ob das nicht ein gegen die Syphilis widerspenstiger Organismus zu sein scheine, und schliesst etwa mit folgenden Sätzen:

1. Wenn ich eine Excision gemacht hätte, hätte ich dieser die Wohlthat einer Verdünnung des Syphilisgiftes nicht zugeschrieben?

2. Wenn ich anderseits eine Auto-Inoculation nach Pontoppidan wiederholt hätte, hätte ich nicht eine benigne Syphilis in eine schwere umgewandelt, mit anderen Worten, darf man unter diesen Umständen nicht eine stärkere Infection (Surinfection) befürchten?

Besnier. Syphilis der Glasbläser. (Paris, G. Masson 1885. Monographie.)

Schon im Jahre 1824 und 1833 war man hestrebt, statt der Glasbläser Jaschinen bei der Glasfabrication einzuführen. Bisher blieb 
es jedoch bei blossen Vorschlägen. Die Uebertragung der Syphilis durch die Rohre veranlasste $1859 \mathrm{Chas}$ aggny Mundstücke für jeden einzelnen Glasbläser zu empfehlen. Vorgebene Mühe! Dio Arbeiter bedienten sich deren nicht. Guinand hat als Fabriksarzt in Rivede-Gier zwei Fälle von syphilitischer Ansteckung durch das Blasrohr constatirt und veranlasst deshalb monatliche Untersuchnngen der Arbeiter. Er untersuchte die Lippen, die Wangen, den Rachen and die Genitalien. Seitdem kam in 9 Jahren nor eine Anstecknng vor. Was die Anstellung syphilitisch erkrankter Bläser anbetrifft, so ist eine Vorsicht bei deren Aufnahme geboton und zwar ist zu beachten, ob der Arbeiter eine zweimonatliche Kur durchgemacht hat, ob die Mund- und Rachenaffecte mindestens einen Monat geschwunden sind, schlirsslich muss or nach seinem Eintritte in die $\Lambda$ rbeit durch zwei Monate hindurch alle 8 Tage und von da an noch 2-3 Monate jeden 15. Tag untersucht werden. Die Sache ist deshalb schwierig, da während der Secundärperiode (2 Jahre hindurch) jede Erosion an der Mundschleimbant die Uebertragung an cinen Gesunden vermittcln kann. Guinand berichtet über bereits bekannte Lippen- und Mandaffectionen, als Einrisse, Excoriationen etc. Neben diesen beobachtet er um den etwas erweiterten Ductus Stenonianus eine Schleimhanterkrankung, die eine Insel bildet von silberweisser Farbe und die nach der von Schwi mmer beschriebenen Leukoplakia buccalis ( $\mathrm{Vid}$ al: Lenkoplasie) benannt werden könntẹ. Das was diese bei don Glas. bläsern speciell vorkommenden Plaques vor anderen auszeichnen soll, ist ihr Sitz um den Duct. Stenon., ihre Symmetrie, ihr Erscheinen zu beiden Seiten für gewöhnlich kleiner als ein Fünf Francs-Stück in Silber und erreichen nur die Commissur, was häufig bei Schleimhautaffectionen (Stomatite épithéliale) aus anderen Ursachen vorzukommen pflegt. Sie entwickeln sich bei Glasbläsern, welche auch Nichtrancher sind, so dass der Tabak auf ihre Entwicklung keinen Einfluss zu haben scheint. Diese Schleimhauterkrankung steht zur Syphilis in. keiner näheren Be\%iehung, denn die Ansteckung bei Glasbläsern kommt an den Lippen, der Zunge und den Mandeln vor.

Tiessier. Hereditäre Syphilis, Leber-und Milzaffection, Osteomalacie. (Annales de derm. et de la syph. T. VI, Nr. 4, p. 207.)

Ein 19jăhriges, in der Entwicklung kanm einem 12jährigen gleichkommendes Individum wird anf die Abtheilung von Dr. Siredey 
aufgenommen. Anamnestisch lässt sich keine für die Syphilis der Eltern sprechende Thatsache vorbringen. Im 6. Lebensjahre wurde beim kleinen Patienten wegen Ascites eine Punction gemacht; ausserdem litt er einmal an Contractur im linken Kniegelenke, welche mit Extension behandelt wurde. Nachdem noch mehrere Erkrankungen die Spitalhilfe nöthig machten, trat vor der Aufnahme eine bedeutende Schwäche der unteren Extremitäten auf. Bei der Aufnahme fand man das Abdomen stark ausgedehnt durch die vergrösserte Leber und die relativ noch grössere Milz. Die unteren Extremitäten abgemagert; die rechte erscheint länger als die linke und in der Diaphyse des Oberschenkels verdickt. Leucocythaemio erscheint ausgeschlossen, da das Verhältniss der rothen zu den weissen Blutkörperchen $300: 1$ beträgt. $\mathrm{Zu}$ diesem Befunde fand man noch eine Verhärtung des Testikels und lineare weissliche Narben an den Lippen und sah sich zur Annahme veranlasst, dass die Ursache der vorgefundenen pathologischen Veränderungen hereditäre Syphilis sei. Die Behandlung (2 Gr. Jodkali p. d. und Eimreibung), konnte der Kranke nicht vertragen. Eine wiederholte Fluctuation am rechten gescliwollenen Oberschenkel machten mehrere Mille Einschnitte nothwendig, bei deren orsterem sich blos blutige Flüssigkeit, bei dem letzteren aber auch blutige Jauche entleerte. Dieser Umstand und ein wiederholt aufgetretener Brechdurchfall machten dem Lriden nach mehr als ein Jahr danernder Behandlung ein Ende. Der Kranke erlag im Collaps.

Bei der Section fand man die Milz 24 Ctm. lang, $1380 \mathrm{Gr}$. schwer; ihre Kapsel stellenweise verdickt, das Parenchym stark verdiehtet; mikroskopisch liess sich pine bindegewebige Entartung der Lymphoiden-Elemente an vielen Stellen nachweisen. Die Leber von normalem Gewichte, zeigte nirgends narbige Einziehungen an der Oberfläche. Mikroskopisch konnte man fast überall die Zunahme des Bindegewebes in annulärer Anordnung (speciell französische Auffassung einzelner Formen der Lebercirrhose; Anmerk. des Referenten.) mit Erbaltung der Leberzellen constatiren. Die Musculatur des Oberschenkels war durchsetzt von Streifen, welche mikroskopisch als eine gummöse Infiltration erschienen. Das darunter liegende Periost überall adhärent verdickt und an der Theilungsstelle der Linea aspera zu einem gummösen Knoten umgewandelt; der Oberschenkelknochen spontan gebrochen, und zwar an einer Stelle, wo der Process der Osteomyelitis gummat. am weitesten gediehen und die compacte 
Knochensubstanz am meisten verdickt war; atsserdem lag an der Bruchstelle quer ein Knochenfragment von $8 \mathrm{Ctm}$., welches noch an Bindegewebsresten mit den übrigen Knochenenden zusammenhing and an dem dig. Knochenresorption am weitesten gediehen war, so dass os wie wurmstichig aussah. In der ganzen Länge des Oberschenkels war das Mark entzündet nnd an zwei noch schärfer umgrenzten Stellen verkäst. Die Tibia der rechten Seite ist in der oberen Partie in ihrem Marke anch vascularisirt und rotl, in der unteren blässer. Die. Gelenke waren frei. Die mikroskopischen Untersuchungen bestätigten den Befund der Ostitis mit Erweiterung der Medullarkanälchen and einer Zellen-Infiltration.

(Anmerkung des Ref. Osteomalacie wird in diesem Falle ein Process genannt, dessen Beschreibung ans klar darthut, dass es sich um eine Ostoonyelitis gummatosa mit consecutiver Veränderung und Knochenbruch gehandelt hat.)

Hermet. Hereditare Syphilis mit Gehorsläsion einhergehend. (Annales de derm. et de la syph. T. VI. Nr. 3. 1885.)

Hermet theilt einen Fall mit, in welchem es sich um eine im 9. Lebensjahre nach einem Schreck binnen vier Tagen aufgetretene Taubheit handelt und bezieht dieselbe auf hereditäre Syphilis, nachdem sie weder hysterischen noch auch tabetischen Ursprungs sein kann und derartige Tanbheit nur diesen drei Ursachen zuzuschreiben ist. Die objective Untersuchung der 25 Jahre altrn Person ergab an dem Gelü̈rorgan einen negativen Befund. Für hereditäre Syphilis sollen Narben an der Cornea, Narben in der linken Achselhöhle, welch let:tere von einer Eröffnung einer Geschwulst herrühren, ausserdem die Anamnese, die den Haupttheil der Krankengeschichte ausmacht, sprechen. Hermet sieht sich zu folgendem Schlusse veranlasst: 1. In gewissen Fällen kann man die hereditäre, syphilitische Diathese blos durch die Untersuchnng der Erscheinungen am Gehörorgane ermitteln; 2. Die tardive, hereditäre Syphilis kann sich allein in Stōrangen des Gelhörorganes kundgeben, die in vollständiger Tanbheit bestehen, rasch auftreten und-sich mit intactem Gehörleitungsapparat vereinigen können. Schliesslich führt Hermet djese Störungen auf eine Neuritis des Gehörorganes zurück. Es gibt somit bei Syphilis Affectionen des Gehörorganes in der ebenerwähnten Form, weiter solche, die als Mittelohrentzündungen anftreten und sich blos durch die Schmerz- 
losigkeit von den gewöhnlichen untérscheiden sollen und endlich eine Form, welche in nachfolgender Krankengeschichte behandelt wird: "Ulceröses Syphilid im äusseren Gehörgange".

Ein dreijähriges, von syphilitischen Eltern abstammendes Mädehen bot mehrere für hereditäre Syphilis sprechende Zuichen; als: eine Nasendeformation, cariöse, kleine Zähne, suspecte Narben am Körper. Seit 7 Monaten hatte das Kind zeitweilig einen Ohrenfluss and ein Geschwür im âusseren Gehörgange und zwar des ganzen unteren Segmentes des Gehörganges mit Zerstörung des unteren Segmentes des Trommelfells. Locale und allgemeine Behandlung brachte das Geschwür in 14 Tagen zur Vernarbung.

Gaudichier. Zwei Beobachtungen, das Colles'sche Gesetz betreffend. (Annales de derm. et de la syphil. T. VI, Nr. 2. 1885.)

Im Verlaufo von einem Jahre beobachtete Gaudichier im Spitale St. Louis nachfolgende zwei Fille, an denen sich das so. genamnte Colles'sche Gesetz bewahrlieitete. Am 15. März $1884 \mathrm{kam}$ in die Abthfilung des Prof. Fournier eine 24jährige Mutter mit ihrem vier Monate alten Kinde, welches mit recenten Syphiliserscheinungen im Gesichte, speciell am Kinn, den Lippen und der Zunge, und auch am übrigen Körper bebaftet war. Das Kind brachte die Eruption im Gesichte mit zur Welt; die übrigen Erscheinungen kamen erst hinzu. Es warde von seiner Matter gestillt, an der sich keine Krankheitssymptome oder deren Reste nachweisen liessen. Die Mutter hat viermal geboren; das erste Kind war ein gesunder Knabe, dann erfolgte zweimal Abortus und als viertes kam das kranke Kind. Die erste Schwangerschaft stammte von einem anderen Manne, die letzten drei von einem, der vor etwa acht Jahren syphilitisch erkrankt war.

Der zweite Fall aus der Abtheilung des Prof. Besnier betrifft ein 7 Wochen altes Kind, welches zallreiche confluirende Papeln im Gesichte mit kleineren Erosionen an der Oberlippe nebst vielen deutlichen syphilitischen Affecten darbietet. Dessen Mutter 21 Jahro alt, war nie mit syphilitischen Ausschlägen etc. behaftet und säugt ihr krankes Kind. Der Vater dieses Kindes hat ebenfalls vor circa acht Jabren Syphilis acquirirt, welche von deutlichen Secundärerscheinungen gefolgt war:

Mraček. 
Joseph. Homiparesis syphil.; Fall intracranieller Syphilis als frühes Recidiv nach Hydr. tann. oxyd. (Aus Köbner's Poliklinik für Hautkrankh. - Berl. klin. Woch. Nr. 30.1885.)

Ein 17jähriger Mann hatte bei Beginn der einem harten Schanker folgenden Hautausschläge und ebenso 6 Wochen später bei Recidiv (zusammen 87) Sublimatinjectionen erhalten, zugleich wurden Inunctionen mit grauer Salbe angewandt. Nach 12jähriger Latenz trat ein unbehandelt gelassener Ausschlag ein, dem 4 Wochen später unter starken Kopfschmerzen in Halbschlaf, starkem Kopfsausen u. A. - eine Lähmung der rechten Körperhälfte folgte. Nach wenigen Stunden liess die Schwäche nach, um bald auf eine geringe bjeibende Paresis bei guter Sensibilität und Temperatursinn, etwas gesteigerten Sehnenreflexen zurückzugehen (Hemiparesis in Folge von Endarteriitis syph. eerebr.) Ausserdem fanden sich an mehreren Stellen annulares papalüses Hautsyphilid und die Inguinal-, Axillar-, Cervical- vul Cubiitaldrüs'n vergrössert und hart. Kōbner weist auf die von ihm jüngst betonte Wichtigkeit der localen Behandlung indurirter Lymphdrüsen hin, die nach der Auffindung des Syphilisbacillus mehr wie je als Depôts von Giftresten verdächtig seien. - Unter Sublimatinjectionen schnelles Abheilen der Syphiliş und Besserung der Motilität.

Ein 42jähriger Mann, der wegen eines sehr grossen indurirten Ulcus and einseitiger grosser und harter Polyadenitis inguinalis während der ersten 3 Monate örtlich 8 mal 15.0 Sap..merc. moll. und dann 18.0 Ungt. ciner. verbraucht hatte, erhielt wegen heftiger dolores osteal. frontis das Lustgarten'sche Quecksilbertannat. In fünf Wochen wurden $10 \mathrm{Grm}$. verbraucht, während welcher Zeit nur geringe Schlemhaut- und Drüsensymptome auftraten, dann durch drei Monate 80 Gr. Jodkaii, dann wieder eine Woche Lustgarten's Präparat. Nach 3 Moniten trat Patient wieder in Behandlung mit linksseitjger Labyrinthaffection und zweifelhafter intercranieller Erkrankung, die theils in das Periost des Schädels, theils in die Gehirnhänte, vielleicht auch in die Gehirnrinde zu verlegen war. (Stupider Gesichtsausdruck enormes continuirliches Kopfreissen, Schwindel, Romberg'sches Symptom, Gang mit gespreizten Beinen, Aphasie, Agraphie, Abnahme der motorischen Kraft beider Beine bei normaler Sensibilität.) Da diese Symptome trot\% vierteljährigen Jodkaliumgebrauches anhielten, wurde zar Schmiercur übergangen, die schnolle Besserung brachte. Köb- 
ner ist durch diesen (und andere) Fälle misstrauisch geworden, ob der dem neuen Präparate nachgerühmte Einfluss auf Verminderung der Recidivfähigkeit der Syphilis bestehe. Caspary.

Charpentier. Gehirnsyphilis und allgemeine Lähmung. (Annal. de derm. et de la syph. T. VI. Nr. 3.)

Ch. theilt einen Fall mit, in dem die betreffende Kranke von der Strasse anfgegriffen warde, da sie ihr Domicil nicht zu finden wusste. Periostitis gummosa frontis, Gumma palpebrae, Ozaena, Otitis media mit Perforation des Trommelfells, partielle Sehnerv-Atrophie nach einer Neuritis, ältere pigmentirte Narben am Perineum und an der grossen rechten Schamlippo sind in Kürze die begleitenden Elscheinungen nachfolgender Lähmungszustände. Die Kranke sitzt halb geliihnt in Sessel, liisst Urin und Stuhl unter sich, auf gestellte Fragen kaun sie nicht antworten, da sie dieselben nicht versteht und bringt nur unverständliche Laute hervor. Die Zunge bleibt zwischen den Zühnen, der Gesichtsausdruck ist unbeweglich, stumpfsinnig; der Speichel fliesst ihr über die herabhängende Unterlippe aus dem Munde; die Nahrung verlangt sie nicht und erwacht nur beim Anbringen derselben an die Lippen; die Arme lässt sie fillen, unterstätzt kasn sie sich mit zitternden Extremitäten bewegen. Die geistigen Störungen und Lähmnngszustände wurden nach Charpentier näher durch die vorerwähnten begleitenden Erscheinungen gekennzeichnet, und man nahm Syphilis als die Ursache derselben an und verordnete $4 \mathrm{Gr}$. Jodkalium p. d. nebst mercuriellen Einreibungen. Nach 14 Tagen besserte sich der Zustand der Kranken, sowohl was die äusseren Zeichen der Syphilis (Gummositäten), als auch was die Motilitätsstörungen betrifft. Der Geisteszustand hat sich nach 4 wöchentlicher Behandlung soweit gebessert, dass die Patientin, wenn auch mühsam, so doch wahrnehmbar nachfolgende Auskünfte geben konnte: Patientin ist 39 Jahre alt, verheiratet und hat einen 17jährigen Sohn; von der Zeit der Ansteckung mit Syphilis, sowie von etwaigen Hautaffectionen weiss die Kranke nichts anzugeben. Sie litt blos ofters an Halsschmerzen und verlor seit einigen Jahren die Hare. Vor sechs Jahren hatte sie Kopfsenmerzen in der Stirne nnd im Hinterhaupte, welche von Ueblichkeiten and Erbrechen gefolgt waren. Vor vier Jahren hatte Patientin den ersten epileptiformen Anfall, der sich dann im letzten Jahre dreimal im Monate wiederholte. Diesen Anfällen gingen keinerlei 
nervöse Erscheinungen voraus. Zar Zeit diešer Angabe ist der Facialis der rechten Seite gelähmt und die rechte obere und untere Extromität paretisch; die Physiognomie gewinnt an Ausdrack. Resumé: Naclı einer Serie epileptiformer Anfälle, welche erst im späteren Alter von 34 Jahren auftraten, verliert die Kranke das Bewusstsein etc, in einer Zeit, wo die tertiären Syphiliserscheinungen äusserlich sich entwickelt haben. Eine rasche Besserung dieser Erscheinungen nebcn der Wiederkehr der intellectuellen und motorischen Fähigkeiten in Folge specifischer Behandlung lässt auf den syphilitischen Ursprung der geistigen und motorischen Störnngen schliessen. Die äusserlich sichtbaren Gummositäten lassen die-Annahme zu, dass es sich um älnliche intracranielle oder meningeale Erkrankungen in diesem Falle liandelt, welche das Bild der von Fournier beschriebenen Psendoparalysie générale syphilitique hervorgebracht haben.

Alexander. Fin Fall von gummösen Geschwülsten der Hirnrinde. (Separat-Abdruck a. d. Breslaner ärztl. Zeitschr. 22. 1884.)

Ein 28jällriger Arbeiter kam am 3. Octob. wegen Nackenstarre, Kopfschmerzen und leichter Somnolenz zur Anfnahme in die Klinik des Prof. Biermer. Anamnese:

Infection vor 6 Jahren; vor 3 Jahren heftige Iritis o. s., zur selben Zeit ein hartnäckiges Fussgeschwür und Hodengeschwulst; vor mehr als 1 Jahre Kopfschmerzen, vor $1 / \%$ Jahren der erste Krampfanfall mit Bewusstlosigkeit, welchem bald immer heftigere und häufigọ auftretende folgten, so dass zur Zeit einer Behandlung wegen Periostealgummen am Thorax in der chirurgischen Klinik jede 2-5 Tage ein epileptischer Anfall vorkam, dem jedoch keine Aura vorausging, spüter aber ein starkes Zitterm in der linken Hand den Beginn des Anfalles anzeigte. Die Kopfschmerzen warden anch immer heftiger, wobei der Kopf stark nach hinten gebengt gehalten wurde. Bcim Bewegen des Kopfes steigerte sich der Schmerz namentlich im Hinterkopfe. Das Gedächtniss des Patienten hat gelitten; die Fragen beantwortet er träge und mit Widerwillen. Die rechte Pupille ist stark verengt (links ist ein Coloboma nach Iridectomie); Bewegungen der Lider, der Angen, der Gesichtsmuskeln und der Zunge sind durchwegs normal; Puls etwas verlangsamt 54 p. Min. Patient kann gehen und stehen, wenn auch unsicher, Patellarreflex beiderseits verstärkt, das Localisationsvermögen sowie. die Tastempfindung der Hant sind. 
erhalten. Die ophthalmoskopische Untersuchung ergibt eine Stauungspapille auf beiden Augen; das Sehvermögen ist erhalten. Der Hirndruck mit Kopfschmerz und Nackensteifheit, die seit 6 Monaten bestehenden epileptischen Anfälle ergaben die Annahme eines intracraniellen Tumors. Nahezu 48 Stunden vor dem Tode stieg die bis nun normale Temperatur auf 38 und $39 \cdot 5^{\circ}$, die Unruhe des Patienten wurde grösser, es stellten sich Delirien ein und Patient starb unter Trachealrasseln bei $39^{\circ} 4^{\circ}$ and $12 \pm$ Pulsschlägen.

Bei der Section fand man eine Osteoporosis syph. am rechten Scheitelbein und Gummata in beiden hinteren Centralwindungen mit Erweichung der darunter liegenden Marksubstanz. Ausserdem Hyperämie und Oedem der Lungen, Hyperämie der Nieren, der Leber und Artcriitis catarrhalis.

In der Epikrise that der Autor dar, dass die meisten Symptomr, als die Benommınheit des Sınsoriums, die Kopfschmerzen, die Staungspapille, die gesteigerten Patellarreflexe sich durch den Befund am Gehim erklären lassen. Die Krampfanfällo sind als Produkt einer Ruizung der motorischen Centra von Seite des Tumors aufzufassen. Die Nackensteifleit dürfte bei dieser intracraniellen Erkrankung entweder in Folge einer reflectorisch tonischen Innervation der Nackenmuskeln durch eine starke Reizung der sensiblen Nerven der Dura oder auch durch eine directe Reizung des Rindencentrums für die Nackenmusculatur zu erklären sein. Letzteres scheint dem Autor nicht wilhrscheinlich.

E. Kohn. Poetische Glossen über den Terminus Psoriasis palmaris et plantaris syphilitica. (Wien. med. Presse 1884.)

$K$. hebt als lobenswerth hervor, dass die jüngeren Autoren äber Syphilidologie die Nomenclatur der Exantheme vereinfachen und wendet sich gegen das Beibehalten der obon angeführten Benennung bei Ausschlägen an der palma manus und planta pedis. Schuppen, Ausscllläge wurden schon von den älteren Autoren genau geschildert, der Ausdruck Psoriasis bei schuppenden, syphilitischen Ausschlägen hat sich erst in den $40 \mathrm{er}$ Jahren eingebürgert.

Die Gründe, welche man für das Fortbestehen des Terminus gewöhnlich anzuführen pflegt, geht der Verf. durch, will aber weder die Aehnlichkeit mit der Psor. vulg. nọch den eigenthümlichen hartnäckigen Verlauf dicser Affection gelten lassen, weil erstere eben nur 
selten und vielleicht nur vorübergehend besteht, letaterer aber anch anderen Syphilisprodukten zukommt. Diese pathologischen Zustände ail der Hohlhand und an der Fusssohle zeigen ihre Eigenthümlichkeiten im Vergleich zu anderen Hautausschlägen bei Syphilis durch die anatom. Beschaffenheit der Haut, an der sie vorkommen, ebenso wie jene in der Plica ani, am Scrotum an der Schleimhautgrenze etc. Verf. meint es gehöre dies nicht zum Wesen der Krankheit, wenn auch der Verlanf ein eigenthümlicher und schleppender ist, denn die Syphilis müsse auf der Haut überall gleiche patholog. Produkte erseugen.

Wenn' auf den Handtellern, Fusssohlen eine Ausschlagsform vorkommt, die als Theilerscheinung einer universellen der Psor. vuly. guttata ähnlichen zu betrachten ist, so ist nach $K$. auch dies kein Grund sie Psoriasis zu nennen, weil bei nüherer Retrachtung die Plizques aus dicht nebeneinander gedrängton schuppenden Papeln bestehen weshalb sie auch besser als Pap. syph. desquamantes bezeichnet werden sollten. Sind demnach neben anderen Flecken, Papeln, Pusteln, solche auf der palina oder planta, so soll man sie ebenfalls so benennen. Erscheint aber ein Exanthem auf den in Rede stehenden Stellen, ohne dass ein ähnliches oder üborhaupt eines anf der übrigen Haut des Körpers nachgewiesen ist, so ist entweder das Exaluthem bereits geschwunden und nur das der palma und planta blieb noch übrig oder das letatere entwickelte sich einige Monate spitter und ist ebenfalls als Theilerscheinung des bereits geschwundenen aufzufassen und darnach za benennen.

Schliesslich kamen in seltenen Fällen oft viele Jahre nach der Infection Erkrankangen der palma oder planta vor, weIche sowohl durch eine besondere oft jahrelange oder durch eine besondere hartnäckige Wiederkehr sich auszeichnen.

Für diese Erkrankung schlägt Verf. vor, den von Fuchs gebrauchten Namen Syphilolepis cornea zu wählen, welcher identisch ist dem. von Biel angegebenen Syplilid squameux corné oder Exanthema syph. tardivum palmare et plantare.

Verf. erwartet, dass eine nähere histologische Untersuchung eine exactere Bezeichnung ergeben liönnte.

Seit Fallopia's Zeiten, führt Verf. des Weiteren aus, haben die Acrzte die Fälle als besonders schwer geschildert, wenn sie die palmae et plantale miterkrankt sahen und haben sich durch diesen 
Irrthum zn falschen Diagnosen und gar vielleicht zu überschwänglichen unnützen, ja sogar schädlichen therapeutischen Massnahmen hinreissen lassen. (Die Erfahrung lehrt aber in der That, dass gerade diese Fälle es sind, bei denen Endarteriitis u. a. cut. Leiden der schwerwiegendsten Art vorzukommen pflegen. Fehler kommen auch obne Psoriasis palm. and plant. vor und werden nicht eher vermieden werden, so lange das schematische Verfahren in der Syphilistherapie nicht aufhören wird.)

Mra ě甘k.

Gaudichier. Syphilitische Phthise, Heilung. (Annales de derm. et de la syphil. T. VI, Nr. 3.)

Am 1. März $1884 \mathrm{kam}$ auf die Abtheilung von Fournier ein Kranker, 39 Jahre alt, der an beiden Unterschenkeln Haut- und Knochengummen trug, deren arste sich bereits vor 23 Jahren gezeigt haben. Die linke Clavicula war auf das Dreifache verdickt. Seit zwei Monaten magerte der Kranke ab, und konnte seiner Beschäftigung nicht nachkommen. Einige Tage vor seinem Eintritte in's Spital hat Pationt erst gehustet, hatte aber Abends kein Firber und keine Schweisse und hat nie Blut ausgeworfen. 2-3 Tage nach der Aufnahmo haben sich reichlich schlẹimig eitrige Sputa mit Striemen ron Blut gezeigt. Der Lungenbefund ergibt eine Caverne in der Spitze der linken Lunge nobst rasselndem Geräusche in dieser and auch der anderen Seite. Fournier stellt somit die Dingnose: syphilitisch, Hyperostosen mit tuberculosem Affecte beider Lungenspitzen, links mehr vorgeschritten. Anamnestisch lässt sich nichts für eine erworbone Syphilis erheben nnd anch die Annahme der hereditären basirt blos auf einer abgelaufenen Otitis chronica ohne Eiterung. 4 Gr. Jodkalium pro die Chinawein, und Verband brachten eine wesentliche Besserung in 14 Tagen sowohl an den Unterschenkeln als auch in den Iungen. Der Kranke erholt s sich, 40 Tage nach seinem Eintritte schwanden nahezu alle pathologischen Erscheinungen an den Lungen und 20 Tage spätér verliess der Kranke das Spital mit vollständig normalem Lungenbefunde. Angesichts der wanderbaren Wirkung, welche hier das Jodkalium hervorbrachte, bestätigt sich die Diagnose auf Syphilis und Gaudichier glaubt es hier auch mit gummöser Erkrankung der Lungenspitzen $z a$ thun gehabt zu haben und schreibt diesem Verfahren in ähnlichen veralteten Fällen von Syphilis und Lungenerkrankungen eine grosse praktische Bedeutung za. Nach 
11 Monaten sah Gaudichier den Kranken wieder, dessen Lungen vollständig geheilt waren. Das Allgemeinbefinden war gat, nur der linke Unterschenkel war nenerdings exulcerirt. Mraček.

Moos und Steinbrügge. Histologischer Beitrag zur Genese der Syphilis-Kachexie. (Berl. klin. Woch. Nr. 19. 1885.)

Moos and Steinbrügge untersuchten die Felsenbeine eines Mannes, der an syphilitischer Caries des Schädels und harten Gaumens, chronischer Pneumonie, amyloider Degeneration in Milz, Leber, Nieren, zu Grunde gegangen war. Späteren ausführlichen Bericht verheissend, berichten sie nur kurz über die Veründerung der Spongiosa in der intra vitam Ruptur der zartwandigen Gefässe des Marks, mithin Untergang zahlloser rother Blutkörperchen stattgefunden habe. Wenn, wie sehr wahrscheinlich sei, derselbe pathologische Vorgang auch in Markräumen anderer Knochen in Zukunft werde nachgewiesen werden, so müsse derselbe zweifellos in hohem Grade störend auf die Blutmischung einwirken und einen wesentlichen Factor bei der Ausbildung der Syphiliskachexie ausmachen, demn zu dem Untergange zahliloser rother Blutkörperchen komme noch die Hemmung der physiologischen Neubildung von Blutkörperchen in Knochenmark hinzu.

Caspary.

Fischer. Syphilitische Necrose des Atlas. Heilung. (D. Ztsch. für Chirargie 20. Bd. 1885.)

7 Monate nach dem Auftreten eines harten Schankers Entwicklung schwerer Halssymptome, Schlingbeschwerden, Hustenreiz, näselnde Sprache, Regurgitiren der Speisen und Getränke durch die Nase. Dabei wurde der Patient immer mehr mager und matt, ein quälender Husten mit eitrigem Auswurf raubte ihm die Nachtruhe, endlich kam es noch zu einem "steifen Nacken". Die Untersuchung der Brust ergab nichts Abnormes, der weiche Gaumen geröthet, dick infiltrirt and derb, unbeweglich und steif. Die hintere Rachenwand in ein grosses grau belegtes Geschwür verwandelt, dessen obere Grenze unbestimmbar, die untere bis zur Znngenbasis reichte. Kehlkopf frei.

Nase und Gehörorgan niclut beeinträchtigt. Jede Drehbewegung des Kopfes beschränkt und schmerzhaft, die Nickbewegungen nur in sehr geringem Masse auszuführen. Schmierkur, Gurgelungen und Aetzungen mit Argent. nitr. lassen eine Besserung im Sprechen und 
bein Schlingen eintreten. Jedoch musste die Schmierkur wegen Salivation schon nach 14 Tagen ausgesetzt werden. Olei jecoris und Jodkali imerlich. - Das Rachengeschwür wird auch besser, nur die Nackensteife persistirt und nimmt noch 2u. Am 7. Februar, also im 10. Krankheitsmonate konnte in der hinteren Rachenwand schon ein entblösster Knochen gefühlt werden. 4 Tage später brachte Patient ein ausgehustetes Knochenstück von $2.5 \mathrm{Ctm}$. Länge, das sich als der vordere Bogen des ersten Halswirbels präsentirte. Die Gelenkflächo für den Zaln des Epistropheus glatt. Jetzt ging die Heilnng sehr rasch vor sich, die Beweglichkeit des Kopfes nach allen Richtungen wurde liergestellt. Keine Recidiven. - Ein ähnlicher Fall, aber im. 12. Jahre der Syphilis ist von Warte 1849 beschrieben worden. Horovitz.

Arcari. Ueber den subcutanen Gebrauch des. Jodnatrium. Vorlesung, gehalten in der Versammlung des Ospedale Maggiore in Mailand, am 14. Februar 1885. (Giorn. ital. delle mal. von. e della pelle. März-April 1885.)

Der Vortragende hat in Fällen, in denen in Folge gastrischer Symptome Jodnatrium innerlich nicht vertragen warde, dasselbe subcutan in Dosen von $0.3-1.0$ in Wasser gelöst injicirt und wurden diese Injectionen, die möglichst tief in die Glutaei eingebracht wurden, vollkommen rnizlos vertragen und zwei solche Injectionen täglich applicirt. Gegenüber dem Syphilisprocess wurden in vielen Fällen sehr gute Resultate erzielt und bei quantitativer Untersuchung des ausgeschiedenen Jod im Urin als auffallend notirt, dass in den Fällen mit günstigem Heileffect die Ausscheidung des Jod langsam und in geringer Menge, umgekehrt in Fällen erfolgloser Behandlung die Ausscheidung des Jod rasch und in grosser Menge erfolgte. Der Verf. schliesst daraus, dass man in den letzteren Fällen grössere Dosen durch längere Zeit geben müsse, um Heileffecte zu erzielen. In Fällen, in denen grosse Dosen Jod indicirt seien, empfehle es sich, neben der innerlichen auch die subcatane Methode anzuwenden, um so den Magen theilweise zu entlasten. Finger.

I. van der Holven. Genesung durch Erysipel. (Nederl. tijdschr. จ. Geneeskunde Nr. 42, 1884. - Centralbl. f. Chir. Nr. 9, 1885.)

Serpiginöse Hautulcera, seit Jahren bestehend, auf beiden Nates und hinterer und innerer Fläche des oberen. Theils des rechten Ober- 
schenkels. Verf. behandelte sie während eines halben Jihres mit verschiedenen internen und externen, specifischen sowie nicht specifischen Mittein resultatlos. Auftreten eines heftigen, spontanen Erysipels, worauf definitive Genesung der Ulcera folgte.

Petichau. Gleichzeitige Behandlang der Syphilis mit Jod innerlich und mit $\mathrm{Hg}$ äusserlich. - Heilung einer schwèren Myelitis. (Arch. med. Belges. 1884.)

P. empfiehlt die interne Verabreichung von Jod und die gleichzeitig vorzunehmende Application von $\mathrm{Hg}$ in Form einer subcutanen Injection. Als Effect dieser combinirten Behandlung tritt rasch Ptyalismus auf. Auf diese Weise gelang es P. einige Formen von Syphilis und eine schwere lurtische Lähmung centraler Natur zn heilen.

Horovitz.

Soffiantini. Beliandlung der constitutionellen Syphilis nach dem Vorgange ron Smirnoff. (Giorn. ital. delle mal. ven. e della pelle. Jänn. Febr. 1885.)

Verf., ein Assistent Scarenzio's, hat 37 Fälle mit subcutanen Calomelinjectionen, zum Theil nach der von Smirnoff angegebenen Methode der tiefen Injectionen in die Glutäi behandelt, gute Resultate erzielt und schliesst sich den von Smirnoff aufgestellten Sätzen an:

1. Dass nach dieser Methode vorgenommen, der in Muskelfleisch cingeschlossene Injectionsherd äusseren Schädlichkeiten wenig zugänglich ist.

2. In Folge der geringen Empfindlichkeit der Musculatur die Schmerzen gering sind.

3. Die Gefahr dor Ulceration wegen der Dicke der Hant- und Muskeldecke gering ist.

4. Doch müsse dio Canüle so lang sein, dass man wirklich in das Muskelfleisch unter die Fascie gelangt.

Finger. 